\title{
La flora exótica ruderal del Parque Nacional La Campana, Región de Valparaíso, Chile central
}

\section{The exotic ruderal flora of La Campana National Park, Valparaíso Region, central Chile}

\author{
Laura Hauck ${ }^{1}$, Andrés Moreira-Muñoz ${ }^{2 *} \&$ Werner Nezadal $^{1}$ \\ ${ }^{1}$ Herbarium Erlangense, LS MPP, Department Biologie, Universität Erlangen-Nürnberg, Staudstr. 5, D 91058, Erlangen, \\ Alemania. \\ ${ }^{2}$ Instituto de Geografía, Pontificia Universidad Católica de Valparaíso, Avenida Brasil 2241, Valparaíso, Chile. \\ *andres.moreira@pucv.cl
}

\section{RESUMEN}

Los ecosistemas mediterráneos, entre los que se cuenta la zona central de Chile, se consideran globalmente entre los más afectados por las invasiones biológicas. Si bien no todas las especies de plantas exóticas se pueden considerar invasoras, en ellas se encuentra el potencial de invasibilidad que eventualmente puede significar cambios sustanciales en la composición de los ecosistemas y las relaciones entre sus especies. Ello es especialmente crítico en espacios protegidos en los cuales se pretende asegurar la conservación presente y futura de las poblaciones de especies nativas y endémicas. En esta investigación se estudió la presencia de plantas exóticas ruderales en los márgenes de los senderos del Parque Nacional La Campana, con el método de transectos. Las familias mejor representadas en la flora exótica ruderal del Parque son Poaceae, Asteraceae y Fabaceae. En cuanto a su forma de vida, el 71\% corresponde a terófitos. La gran mayoría (92,7\%) es de origen euroasiático-norafricano. Al examinar la presencia de las plantas exóticas ruderales en las diferentes unidades de vegetación se obtiene que el Puyal con suculentas es la unidad más afectada. En cambio, las unidades a mayor altitud como el Bosque caducifolio y el Matorral altoandino son las que muestran una menor presencia de especies introducidas. Hay dos factores que parecen ejercer una influencia importante en el grado de invasibilidad: la cantidad de luz disponible y la altitud a la que se encuentra la unidad de vegetación. Para poder evaluar la tasa de expansión y los posibles efectos sobre las especies nativas y el ecosistema del PN La Campana, conviene diseñar un plan de monitoreo, sobre todo de las especies que están más difundidas en el Parque y que aparecen sistemáticamente en los muestreos en distintos tipos de vegetación.

Palabras clave: Plantas exóticas, biogeografía de invasiones, ecosistema mediterráneo, amenazas a la biodiversidad.

\begin{abstract}
Central Chile belongs to one of the world's Mediterranean ecosystems, which are especially prone to biological invasions. Although not all exotic plants can be considered as invaders, they contain an invasion potential that can drastically modify native ecosystems and the relationships between species. This is especially relevant in protected areas that have the explicit mission of biodiversity conservation of native and endemic species through time. The presence of ruderal exotic plants in La Campana National Park, central Chile, was evaluated by means of the transect method applied to both sides of each pathway. The best-represented families in the exotic flora of the park are Poaceae, Asteraceae and Fabaceae. In terms of life form, 71\% are therophytes. The vast majority (92.7\%) are of Euroasian-North African origin. Considering the presence of exotic plants within specific vegetation units, the Puyal con suculentas is the most affected type. In contrast, introduced species were sparse in both the deciduous forest and the alpine scrub. Two factors seem to have an important influence on the potential of the invasion: the amount of available light and the altitude of the vegetation unit. To evaluate the rate of expansion and possible effects on native species and the whole ecosystem of La Campana National Park, a monitoring plan should be designed, especially towards species that are more widespread in the park and appear systematically in the sampling within different vegetation types.
\end{abstract}

KEYwORDs: Exotic plants, biogeography of invasions, Mediterranean ecosystems, threats to biodiversity. 


\section{INTRODUCCIÓN}

Las especies invasoras se han transformado en los últimos decenios en un tema clave en biogeografia, ecología y evolución, y en un problema de alcance global (Pauchard et al. 2016). El comercio internacional y el turismo se cuentan entre las principales causas del constante incremento en las tasas de intercambio biótico mediado por el ser humano, entre regiones del planeta separadas por extensas barreras geográficas, como océanos y grandes cadenas montañosas (Sax 2002, Weber \& Joshi 2009, Pauchard et al. 2011). Las implicancias de este intercambio biótico son inconmensurables, abarcando aspectos ecológicos, económicos e impactos directos en la calidad de vida y salud humanas. En las comunidades bióticas, las especies invasoras son capaces de modificar los patrones de abundancia y distribución de las especies nativas, y muchas veces, son causantes de la extinción de estas últimas (Williamson 1996, Jaksic \& Castro 2014). Como especies invasoras, las plantas vasculares se cuentan entre las más omnipresentes de la biosfera, y una gran parte ha sido expresamente introducida para uso agrícola, silvícola, con fines ornamentales o medicinales; también muchas especies han sido introducidas accidentalmente (Williamson 1996, Newsome \& Noble 1986). Los ecosistemas mediterráneos se encuentran entre los más afectados por las especies introducidas. Se trata especialmente de especies ruderales de borde de ambientes agrícolas, y a lo largo de senderos dentro de los espacios rurales (Kowarik 2003). Para Chile se han reportado recientemente entre 740 y 860 especies de plantas exóticas naturalizadas (Fuentes et al. 2013, Ray et al. 2014); la mayor parte de ellas se concentra en Chile mediterráneo (Jiménez et al. 2008). Globalmente, los ecosistemas mediterráneos cubren apenas un $5 \%$ de la superficie terrestre y contienen cerca del $20 \%$ de la riqueza de plantas vasculares (Cowling et al. 1996). Es por ello que se consideran como hotspots o centros de diversidad botánica global (Davis et al. 1997, Mittermeier et al. 2004). También son considerados como centros globales de endemismo (Vanderplank et al. 2014). Al mismo tiempo, los ecosistemas Mediterráneos son los más amenazados por las especies invasoras, debido a su larga historia de ocupación humana, un clima propicio al establecimiento de plantas, y posiblemente una condición de base de afinidad filogenética (Figueroa et al. 2004, Castro \& Muñoz-Schick 2006, Pauchard et al. 2011). Chile Mediterráneo cuenta aproximadamente con 2.500 especies nativas de las cuales un $48 \%$ corresponde a especies endémicas (Vanderplank et al. 2014). Se desconoce el número exacto de especies introducidas, así como su grado de invasibilidad potencial; prospecciones recientes en la Cordillera de la Costa de la Región de Valparaíso arrojan la presencia de más de 320 especies alóctonas naturalizadas (Teillier et al. 2010), las que en ciertos sitios pueden alcanzar altos valores de cobertura, cercanos al $70 \%$.

A pesar de la importancia de las invasiones en los ecosistemas de tipo Mediterráneo, aún es limitado el conocimiento en cuanto a las causas subyacentes a las invasiones, los procesos históricos, y sus consecuencias para las especies nativas y las funciones ecosistémicas (Jaksic \& Castro 2014). La gran invasibilidad de las especies exóticas herbáceas se evidencia en Chile mediterráneo en casi todos los terrenos agrícolas o perturbados; en éstos la vegetación herbácea nativa ha sido sustituida en gran medida por plantas herbáceas ruderales europeas (Reiche 1903, Arroyo et al. 2000). Sin embargo, no sólo las áreas urbanas, agrícolas y forestales han sido invadidas; incluso las áreas protegidas están afectadas por las especies exóticas a través de caminos y senderos de visita, lo cual en el largo plazo podría afectar la riqueza de las especies nativas (Jiménez et al. 2013). Usualmente el establecimiento de las especies exóticas data de antes de la declaración como zona protegida, y en muchos casos, aún hoy no se logra restringir el acceso del ganado a estas áreas. El impacto de las invasiones de plantas en áreas protegidas del centro-sur de Chile es muy importante, habiéndose detectado altos niveles de homogeneización biótica (Pauchard et al. 2013). A pesar del reconocimiento de la importancia de la información georreferenciada acerca de la presencia de especies exóticas en Chile, es aún escasa la información concreta disponible para áreas protegidas (pero ver Mardones et al. 2015), realidad compartida con muchos países de América Latina (Gardener et al. 2011).

Esta investigación tiene como objetivo evaluar en qué medida el Parque Nacional La Campana está afectado por la presencia de especies exóticas, y específicamente cuáles son las unidades de vegetación más afectadas por estas especies. Se asume que los senderos son corredores funcionales para la dispersión de especies de plantas introducidas en las áreas protegidas (Pauchard \& Alaback 2004). Por lo tanto, se registró la existencia y dominancia de especies exóticas en los márgenes de senderos, en los diferentes tipos de vegetación del Parque. Además se analizó si la presencia de las especias se limita a los márgenes de los senderos o si son capaces de establecerse y extenderse hacia el interior de las unidades de vegetación (Vidal 2005). El presente trabajo no pretende ser por lo tanto un catálogo exhaustivo de la flora introducida del Parque Nacional La Campana, que podría alcanzar el centenar de especies (Geomanejo Consultores 2012). Prospecciones de largo plazo, en distintas épocas y al interior de las unidades de vegetación, podrán completar esta tarea aún pendiente para un gran número de áreas protegidas (Quiroz et al. 2009). 


\section{MATERIALES Y MÉTODOS}

UBICACIÓN

El Parque Nacional la Campana constituye una de las áreas protegidas más importantes dentro de la Ecorregión del Bosque Esclerófilo (Gajardo 1994, Moreira-Muñoz \& Troncoso 2014). Debido al temprano reconocimiento de sus valores biológicos y especialmente botánicos, fue declarado como Parque Nacional el año 1967 y como Reserva de la Biosfera en 1984 (Elórtegui \& Moreira-Muñoz 2009). El Parque Nacional La Campana se encuentra en la Cordillera de la Costa de Chile central entre $32^{\circ} 55^{\prime}-33^{\circ} 01^{\prime}$ 'S y $71^{\circ} 09^{\prime}$ $71^{\circ} 01^{\prime} \mathrm{O}$ y ocupa tres cuencas principales: Ocoa, Cajón Grande, y Granizo, que están separadas por el cordón de cerros que une las cimas de los cerros La Campana (1.828 m s.n.m.) y El Roble (2.222 m s.n.m.) (Fig. 1).
El clima corresponde al de tipo Mediterráneo, de inviernos lluviosos y templados, con veranos secos y cálidos. La temperatura media es de $18{ }^{\circ} \mathrm{C}$ pero la influencia de la corriente de Humboldt en la costa occidental de Sudamérica hace que la temperatura se reduzca en aproximadamente $4{ }^{\circ} \mathrm{C}$ (Pliscoff 2009). El relieve es de gran importancia para las condiciones climáticas locales: las precipitaciones pueden llegar a $400 \mathrm{~mm}$ anuales en el lado de barlovento, mientras que en el lado de sotavento alcanzan poco más de $300 \mathrm{~mm}$ anuales (Pliscoff 2009). Además de eso, la neblina costera que se desarrolla entre 300 y $800 \mathrm{~m}$ de altitud es un factor climático importante. La neblina reduce considerablemente la radiación solar, y aporta directamente humedad al ecosistema por la condensación de agua en laderas empinadas (Pliscoff 2009).

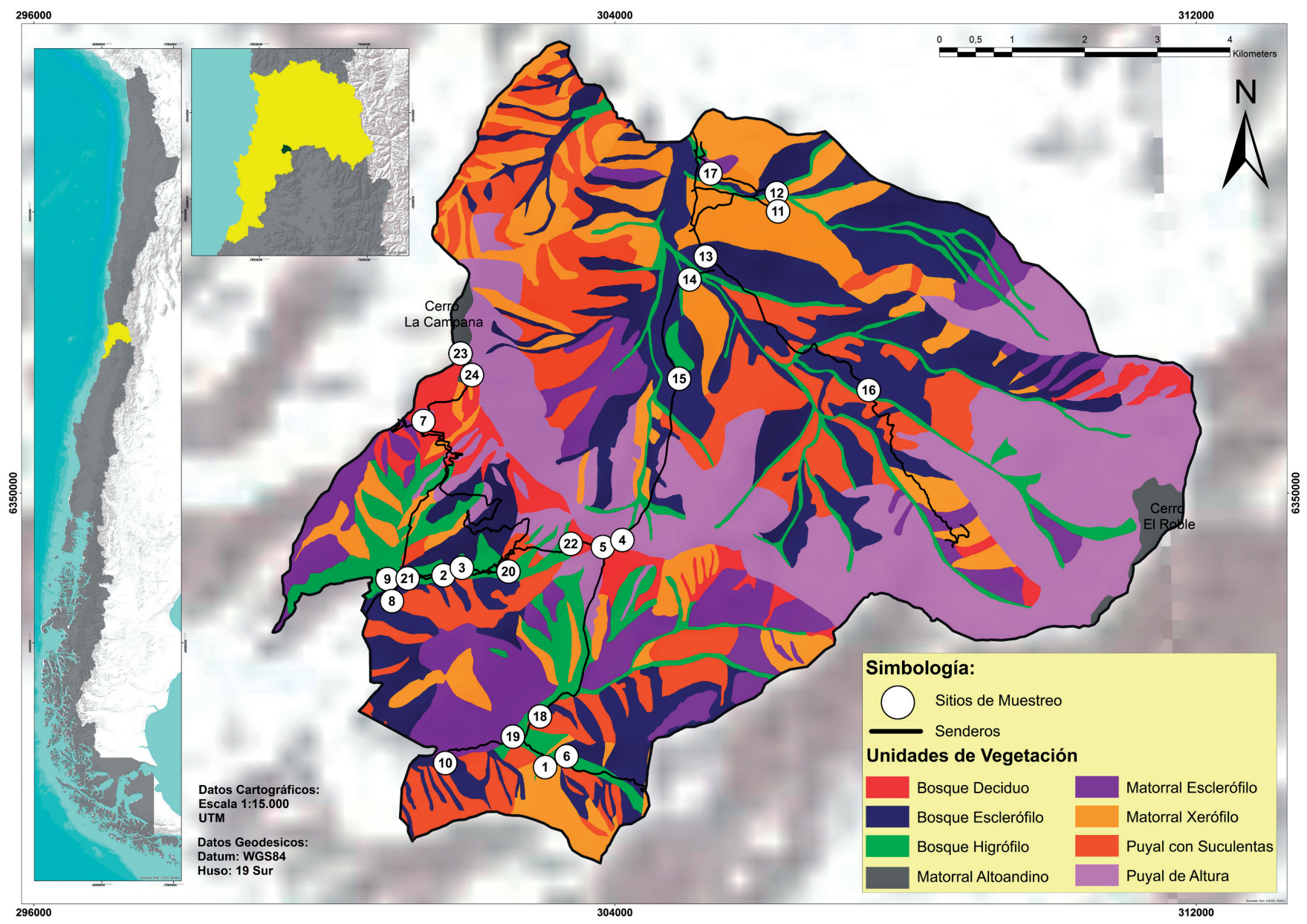

Figura 1. Unidades de vegetación, senderos y sitios de muestreo en el Parque Nacional La Campana. Mapa modificado de Luebert et al. (2009). El Bosque Higrófilo se encuentra prácticamente restringido a los fondos de quebrada.

Figure 1. Vegetation units, trails and sample sites in La Campana National Park. Map adapted from Luebert et al. (2009). Higrophylous forest is almost restricted to the deep valleys and quebradas. 
Flora exótica ruderal del Parque Nacional La Campana: HAucK, L. ET AL.

TABla I. Unidades de vegetación prospectadas en el Parque Nacional La Campana (modificado Luebert et al. 2009).

TABLE I. Vegetation units prospected in the La Campana National Park (adapter from Luebert et al. 2009).

\begin{tabular}{|c|c|c|}
\hline & Unidad de vegetación & Ubicación y características \\
\hline 1 & Bosque esclerófilo & $\begin{array}{l}\text { En laderas de exposición sur/oeste, de } 350 \text { a } 1.050 \text { msnm. 'Bosque esclerófilo de Cryptocarya } \\
\text { alba y Peumus boldus' }\end{array}$ \\
\hline 2 & Matorral esclerófilo & $\begin{array}{l}\text { En mayores laderas de exposición sudeste/oeste, noroeste/este; de } 500 \text { a } 1.050 \text { msnm. 'Bosque } \\
\text { esclerófilo de Quillaja saponaria y Lithrea caustica'; muestra a menudo aspectos de matorral. }\end{array}$ \\
\hline 3 & Bosque higrófilo & $\begin{array}{l}\text { En fondos de quebrada. 'Bosque laurifolio higrófilo de Beilschmiedia miersii' (con Dasyphyllum } \\
\text { excelsum y Cryptocarya alba) y 'Bosque laurifolio higrófilo de Drimys winteri', acompañado de } \\
\text { Crinodendron patagua, Otholobium glandulosum y Escallonia myrtoidea. }\end{array}$ \\
\hline 4 & Matorral xerófilo & $\begin{array}{l}\text { En laderas de exposición norte y noroeste; entre } 400 \text { y } 1.000 \text { msnm. 'Matorral de Retanilla } \\
\text { trinervia' }\end{array}$ \\
\hline 5 & Puyal con suculentas & $\begin{array}{l}\text { En laderas de exposición norte, noreste y este; entre } 450 \text { y } 1.100 \text { msnm. 'Matorral de Puya } \\
\text { berteroniana y Trichocereus chiloensis': vegetación abierta con suculentas. Entre } 1.000 \text { y } 1.300 \\
\text { msnm: 'Matorral de Gochnatia foliolosa con Solenomelus pedunculatus'. }\end{array}$ \\
\hline 6 & Puyal de altura & $\begin{array}{l}\text { En empinadas laderas norte y noreste, entre } 1.100 \mathrm{msnm} \text { y } 1.300 \mathrm{msnm} \text {. 'Matorral de Puya } \\
\text { caerulea': con una estrata arbustiva y herbácea baja y abierta. }\end{array}$ \\
\hline 7 & Bosque caducifolio & $\begin{array}{l}\text { En laderas de exposición Sur a partir de } 1.100 \mathrm{msnm} \text {. Bosque denso de robles o 'Bosque } \\
\text { caducifolio de Nothofagus macrocarpa y Ribes punctatum', hasta los } 1.500 \mathrm{msnm} \text {. En } \\
\text { pendientes empinadas y con menor cobertura, sobre } 1.500 \mathrm{msnm} \text { : 'Bosque caducifolio de } \\
\text { Nothofagus macrocarpa con Schizanthus hookeri'. }\end{array}$ \\
\hline 8 & Matorral altoandino & $\begin{array}{l}\text { Vegetación andina creciendo en las cimas del Cerro La Campana y Cerro El Roble. Fisionomía } \\
\text { de arbustos acojinados, con la presencia de Mulinun spinosum, Chuquiraga oppositifolia, } \\
\text { Tetraglochin alatum y Laretia acaulis. }\end{array}$ \\
\hline
\end{tabular}

La flora nativa del Parque Nacional La Campana está compuesta de aproximadamente 430 especies (Geomanejo Consultores 2012). La familia más diversa es la de las Asteráceas, con predominancia de especies de los géneros Senecio, Haplopappus, Baccharis y Chaetanthera. Otros géneros nativos muy bien representados en el Parque son Calceolaria, Adesmia y Oxalis.

El nivel de endemismo botánico del Parque es muy alto: posee alrededor de 250 especies endémicas de Chile, es decir, sobre un 50\% de especies endémicas (Geomanejo Consultores 2012). Incluso debe ser una de las unidades con mayor presencia de endemismos locales, tales como Calceolaria campanae Phil. (Calceolariaceae); Erigeron campanensis H. Valdebenito, T.K. Lowrey et Stuessy (Asteraceae), Senecio garaventai Cabrera (Asteraceae), Pyrrhocactus garaventai (F. Ritter) F. Ritter (Cactaceae); Ribes quillotense P. Hechenleitner (Grossulariaceae), y Oxalis campanensis Lourteig (Oxalidaceae) (MoreiraMuñoz \& Salazar 2014).

La vegetación del Parque consiste en un complejo mosaico vegetal asociado a distintas situaciones de exposición y pendientes. Las comunidades presentan tanto situaciones típicas dentro de la Región del Matorral y del
Bosque Esclerófilo (Gajardo 1994), como situaciones topoclimáticas y edáficas más específicas, tratadas como variantes en la descripción de la vegetación del Parque (Luebert et al. 2009). Para efectos de la presente investigación se ha realizado una adaptación simplificada de la clasificación de Luebert et al. (2009) (Tabla I, Fig. 1):

El bosque y matorral esclerófilo es la comunidad que concentra el mayor número de especies nativas y endémicas (Villaseñor 1998). Las especies leñosas exóticas más frecuentes en los sectores bajos y cerca de los sitios de acampar son la zarzamora (Rubus ulmifolius Schott), la rosa mosqueta (Rosa moschata Hermann), y el peumo europeo (Crataegus monogyna Jacq.) (Teillier et al. 2003). Sin embargo, aún existe un limitado conocimiento acerca de la flora exótica ruderal herbácea.

\section{REGISTRO DE ESPECIES EXÓTICAS}

Para el registro de las especies exóticas en los márgenes de senderos se realizaron dos a cuatro transectos de entre 20 y 40 m en cada una de las unidades de vegetación (Fig. 1). En las unidades de vegetación Bosque caducifolio y Matorral altoandino se llevó a cabo sólo un registro a causa de su escasa extensión en el Parque. Para el registro 
del Matorral altoandino se eligió un área de 5 por $5 \mathrm{~m}$. La estimación de la frecuencia de las especies se realizó según la escala de Braun-Blanquet (Tremp 2005). Los senderos del Parque La Campana poseen un ancho de entre 1 y 3 $\mathrm{m}$, y son transitados por visitantes, y ocasionalmente por arrieros con algunas cabezas de ganado. Es por ello que la flora de márgenes de senderos tiene características de una flora ruderal (Vidal 2005, Casado et al. 2015).

Se evaluó la capacidad de las plantas exóticas de penetrar desde los márgenes de los senderos hacia el interior (Tremp 2005), para lo cual se realizó muestreos a 2 y $5 \mathrm{~m}$ alejados del sendero. A causa de la vegetación demasiado densa en la unidad de Matorral xerófilo, en este caso se investigó sólo hasta $2 \mathrm{~m}$ del sendero.

La identificación taxonómica, origen geográfico y formas de vida de las especies se realizó según Navas (1973, 1976, 1979), Hohenester \& Welss (1993), Matthei (1995), Hoffmann (1998), Oberdorfer (2001) y Teillier et al. (2010). Fue utilizado además como fuente el "Herbari Virtual del Mediterrani Occidental" del Àrea De Botànica, Department De Biologia, Universitat De Les Illes Baleares y el Herbario SGO. La pertenencia a un cierto elemento florístico europeo se analizó también con las mismas fuentes.

ANÁLISIS

Sobre la base de los datos del muestreo de vegetación se analizó cuáles son las especies exóticas que aparecen con mayor frecuencia, cuántos metros pueden penetrar desde los márgenes de los senderos hacia el interior y con qué frecuencia están representadas en el Parque.

Para evaluar qué especies se encuentran en un estadio avanzado de integración con la flora nativa del Parque, las especies fueron ordenadas según su presencia absoluta relacionada con el número total de recolecciones en las unidades prospectadas. Las especies que tienen la misma presencia absoluta han sido ordenadas según el número de unidades de vegetación en que crecen (Tabla III).

Si se conoce el número de especies que aparecen o desaparecen tras la transición al sitio se puede deducir la capacidad de una especie de penetrar desde el margen del sendero hacia el interior. Por lo tanto se usó la tasa de recambio (Tr) para investigar el cambio de la composición de la flora exótica ruderal en la transición del margen del sendero al sitio a $2 \mathrm{~m}$ de distancia y en la transición de este punto al sitio a $5 \mathrm{~m}$ de distancia:

$\mathrm{T}_{\mathrm{r}}=\left({ }^{+} \mathrm{A}_{\mathrm{n} 1 ; \mathrm{n} 2}+{ }^{-} \mathrm{A}_{\mathrm{n} 1 ; \mathrm{n} 2}\right)\left(\mathrm{n}_{1}+\mathrm{n}_{2}\right)^{-1}$

${ }^{+} \mathrm{A}_{\mathrm{n} 1 ; \mathrm{n} 2}=$ número de especies nuevas que aparecen en la transición del sitio 1 al sitio 2

$-\mathrm{A}_{\mathrm{n} 1 ; \mathrm{n} 2}=$ número de especies que desaparecen con la transición

$\mathrm{n}_{1}=$ número de especies en sitio 1

$\mathrm{n}_{2}=$ número de especies en sitio 2

\section{RESULTADOS}

CONFORMACIÓN DE LA FLORA EXÓTICA

En el área de estudio, los senderos del Parque Nacional La Campana, se registraron 59 especies alóctonas (Tabla II). Las especies exóticas identificadas pertenecen a 17 familias. De ellas, Poaceae, Asteraceae y Fabaceae son las mejor representadas. Este hecho coincide con los resultados del estudio de Teillier et al. (2010) sobre la flora exótica de la cordillera de la Costa a la latitud de Valparaíso, y con los trabajos de Pauchard et al. (2013) y Jiménez et al. (2013). Las especies pertenecen a 47 géneros diferentes, de los cuales Galium, Bromus y Trifolium son los mejor representados, con tres especies cada uno (Tabla II).

ORIGEN GEOGRÁFICO DE LAS ESPECIES EXOTICAS

Un 92,7\% de las especies exóticas del Parque son de origen euroasiático-norteafricano. Sólo cuatro especies tienen su área de distribución fuera de Europa, teniendo en cuenta que muchas de las especies analizadas se consideran hoy en día cosmopolitas (Fig. 2). El origen de Cotula australis, Cynodon dactylon y Matricaria discoidea no se pudo determinar con toda certeza. El predominio de especies exóticas de origen euroasiático-norteafricano concuerda con los conocimientos previos existentes sobre la flora exótica de Chile continental. Arroyo et al. (2000) señalan Eurasia y África del Norte como el área de origen de un $71,2 \%$ de las especies no-nativas de Chile continental. También Jiménez et al. (2008) reportan Europa como la fuente principal de especies introducidas tanto en Chile central como en California.

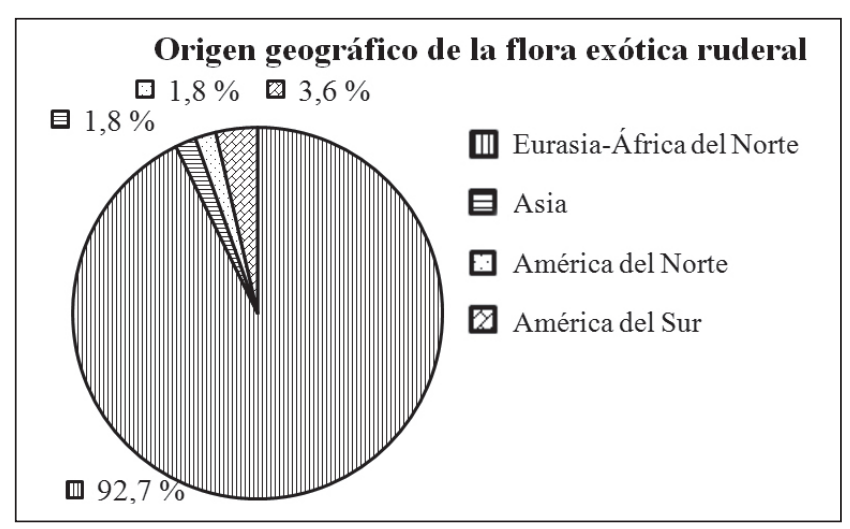

Figura 2. Origen geográfico de la flora exótica ruderal del Parque Nacional La Campana.

Figure 2. Geographic origin of the exotic ruderal flora of $\mathrm{La}$ Campana National Park. 
Flora exótica ruderal del Parque Nacional La Campana: HAucK, L. ET AL.

TABla II. Las especies exóticas ruderales del Parque Nacional La Campana ordenadas por familia y según origen geográfico o elemento florístico, extensión actual y la forma de vida según Raunkiaer. atl: atlántico; euras: eurasiático; med: mediterráneo; no: nórdico; pantrob: pantrópico; smed: submediterráneo; subatl: subatlántico; subocean: suboceánico; subtrop: subtrópico; wmed: mediterráneo-oeste.

TABLE II. Exotic ruderal species from La Campana National Park, sorted by family and including the geographic origin / floristic element, current distribution, and life form according to Raunkiaer. atl: Atlantic; euras: Euroasiatic; med: Mediterranean; no: Nordic; pantrob: Pantropical; smed: Submediterranean; subatl: Subatlantic; subocean: Suboceanic; subtrop: Subtropical; wmed: West Mediterranean.

\begin{tabular}{|c|c|c|c|}
\hline ESPECIE & FAMILIA & $\begin{array}{l}\text { ORÍGEN GEOGRÁFICO [ELEMENTO FLORÍSTICO] - } \\
\text { EXTENSIÓN ACTUAL }\end{array}$ & FORMA DE VIDA \\
\hline Anthriscus caucalis M. Bieb. ${ }^{(1)}$ & Apiaceae & Europa $^{(1)}\left[\right.$ smed $\left.^{(2)}\right]$ - naturalizada mundialmente ${ }^{(2)}$ & terófito $^{(2)}$ \\
\hline Conium maculatum L. ${ }^{(1)}$ & Apiaceae & $\begin{array}{l}\text { Europa }^{(1)}\left[\text { smed-euras }(\text { subocean })^{(2)}\right] \text { - en zonas } \\
\text { templadas }^{(2)} ; \text { cosmopolita }^{(1)}\end{array}$ & $\begin{array}{l}\text { hemicriptófito } \\
\text { (terófito) }^{(2)}\end{array}$ \\
\hline Torilis nodosa (L.) Gaertner ${ }^{(1)}$ & Apiaceae & Mediterráneo europeo ${ }^{(1)}\left[\operatorname{med}^{(2)}\right]$ & terófito $^{(2)}$ \\
\hline Anthemis cotula L. ${ }^{(1)}$ & Asteraceae & $\begin{array}{l}\text { Europa }^{(1)}[\text { med-smed-euras-subocean } \\
\left.\text { templadas }^{(2)}\right] \text { - en zonas } \\
\text { cosmopolita }^{(6)}\end{array}$ & terófito $^{(2)}$ \\
\hline Bidens subalternans DC. ${ }^{(1)}$ & Asteraceae & Sudamérica $^{(1)}$ & terófito $^{(1)}$ \\
\hline Carduus pycnocephalus L. ${ }^{(1)}$ & Asteraceae & Europa $^{(1)}\left[\mathrm{med}^{(6)}\right]$ & $\begin{array}{l}\text { hemicriptófito / } \\
\text { terófito }^{(3)}\end{array}$ \\
\hline Carthamus lanatus L. ${ }^{(1)}$ & Asteraceae & Europa $^{(1)}\left[\operatorname{med}^{(2)}\right]$ & terófito $^{(2)}$ \\
\hline $\begin{array}{l}\text { Cotula australis (Sieb. ex Sprengel) } \\
\text { Hook.f. }^{(1)}\end{array}$ & Asteraceae & Nueva Zelanda ${ }^{(1)}$ o Sudáfrica ${ }^{(6)}$ & terófito ${ }^{(4)}$ \\
\hline Logfia gallica (L.) Coss. \& Germ. ${ }^{(1)}$ & Asteraceae & Europa $^{(1)}\left[\right.$ med-atl $\left.{ }^{(2)}\right]$ & terófito ${ }^{(2)}$ \\
\hline Galinsoga parviflora Cav. ${ }^{(1)}$ & Asteraceae & Perú $^{(1)}$ - cosmopolita ${ }^{(1)}$ & terófito ${ }^{(2)}$ \\
\hline Hypochaeris glabra L. ${ }^{(2)}$ & Asteraceae & Europa $^{(2)}\left[\right.$ subatl-smed-med $\left.{ }^{(2)}\right]$ & terófito ${ }^{(2)}$ \\
\hline Hypochaeris radicata L. ${ }^{(1)}$ & Asteraceae & $\begin{array}{l}\left.\text { Europa }^{(1)}[\text { subatl(-smed })^{(2)}\right] \text { - en zonas oceánicas- } \\
\text { frías-templadas }\end{array}$ & hemicriptófito $^{(2)}$ \\
\hline Matricaria discoidea DC. ${ }^{(1)}$ & Asteraceae & $\begin{array}{l}\text { Nordeste de } A \operatorname{sia}^{(2)} \mathrm{o} \text { eventualmente Noroeste de } \\
\text { América }^{(1 / 2)}-\text { en zonas frías-templadas, naturalizada } \\
\text { mundialmente }^{(2)}\end{array}$ & terófito ${ }^{(2)}$ \\
\hline Senecio vulgaris L. ${ }^{(1)}$ & Asteraceae & Europa $^{(1)}\left[\right.$ med-euras $\left.{ }^{(2)}\right]-$ cosmopolita $^{(1)}$ & $\begin{array}{l}\text { (hemicriptófito) } \\
\text { terófito }^{(2)}\end{array}$ \\
\hline Sonchus oleraceus L. ${ }^{(1)}$ & Asteraceae & $\begin{array}{l}\text { Europa }^{(1)}\left[\text { euras-smed-med }^{(2)}\right] \text { - zonas templadas } \\
\text { cosmopolita }^{(2)}\end{array}$ & $\begin{array}{l}\text { Hemicriptófito } \\
\text { (terófito) }^{(2)}\end{array}$ \\
\hline Cynoglossum creticum Mill. ${ }^{(1)}$ & Boraginaceae & Europa del Sur ${ }^{(1)}\left[\operatorname{med}^{(3)}\right]$ & hemicriptófito ${ }^{(3)}$ \\
\hline $\begin{array}{l}\text { Capsella bursa-pastoris (L.) Medik. } \\
\text { (2) }\end{array}$ & Brassicaceae & Mediterráneo $^{(3)}\left[(\mathrm{w}) \mathrm{med}^{(2)}\right]$ & $\begin{array}{l}\text { hemicriptófito / } \\
\text { terófito }^{(3)}\end{array}$ \\
\hline Cardamine hirsuta L. ${ }^{(1)}$ & Brassicaceae & Europa $^{(1)}\left[\right.$ subatl-smed $\left.^{(2)}\right]$ & terófito $^{(2)}$ \\
\hline Sisymbrium officinale (L.) Scop. ${ }^{(1)}$ & Brassicaceae & Europa $^{(1)}\left[\right.$ euras-smed $\left.{ }^{(2)}\right]$-zonas frías-templadas ${ }^{(2)}$ & terófito ${ }^{(2)}$ \\
\hline Cerastium glomeratum Thuill. ${ }^{(1)}$ & Caryophyllaceae & $\begin{array}{l}\text { Europa }^{(1)}[\text { euras-subocean } \\
\\
\left.\text { cosmopolita }^{(6)}\right] \text { - zonas templadas }\end{array}$ & $\begin{array}{l}\text { (hemicriptófito) } \\
\text { terófito }^{(2)}\end{array}$ \\
\hline Polycarpon tetraphyllum (L.) L. ${ }^{(1)}$ & Caryophyllaceae & $\begin{array}{l}\text { Mediterráneo europeo }{ }^{(1)}\left[\text { med-smed }^{(2)}\right]-\text { zonas } \\
\text { cálidas-templadas }^{(2)} ; \text { cosmopolita }^{(1)}\end{array}$ & terófito ${ }^{(2)}$ \\
\hline Silene gallica L. ${ }^{(1)}$ & Caryophyllaceae & Europa $^{(1)}\left[\operatorname{med}^{(2)}\right]$ - en zonas cálidas, cosmopolita ${ }^{(2)}$ & terófito $^{(2)}$ \\
\hline Stellaria media (L.) Vill. ${ }^{(1)}$ & Caryophyllaceae & $\begin{array}{l}\text { Europa }^{(1)}[\text { no-euras-med } \\
\\
\left.\operatorname{cosmopolita}^{(2)}\right] \text { - zonas templadas }\end{array}$ & terófito ${ }^{(2)}$ \\
\hline Euphorbia peplus L. ${ }^{(1)}$ & Euphorbiaceae & $\begin{array}{l}\text { Europa }^{(1)}[\text { med-smed-subatl } \\
\text { templadas }^{(2)} ; \text { cosmopolita }^{(6)}\end{array}$ & terófito ${ }^{(2)}$ \\
\hline
\end{tabular}




\begin{tabular}{|c|c|c|c|}
\hline ESPECIE & FAMILIA & $\begin{array}{l}\text { ORÍGEN GEOGRÁFICO [ELEMENTO FLORÍSTICO] - } \\
\text { EXTENSIÓN ACTUAL }\end{array}$ & FORMA DE VIDA \\
\hline Medicago arabica (L.) Huds. ${ }^{(1)}$ & Fabaceae & Mediterráneo europeo ${ }^{(1)}\left[\right.$ med-atl $\left.{ }^{(2)}\right]$ & terófito ${ }^{(2)}$ \\
\hline Medicago polymorpha L. ${ }^{(1)}$ & Fabaceae & $\begin{array}{l}\text { Mediterráneo europeo }{ }^{(1)}\left[\operatorname{med}^{(2)}\right] \text { - naturalizada } \\
\text { mundialmente }\end{array}$ & terófito ${ }^{(2)}$ \\
\hline Trifolium dubium Sibth. ${ }^{(1)}$ & Fabaceae & Europa $\left.^{(1)}[\text { subatl(-smed) })^{(2)}\right]$ - zonas frías-templadas ${ }^{(2)}$ & terófito $^{(2)}$ \\
\hline Trifolium glomeratum L. (1) & Fabaceae & Europa $^{(1)}\left[\right.$ med-atl $\left.{ }^{(6)}\right]$ & terófito $^{(3)}$ \\
\hline Trifolium tomentosum L. ${ }^{(1)}$ & Fabaceae & Europa $^{(1)}\left[\mathrm{med}^{(6)}\right]$ & $\begin{array}{l}\text { hemicriptófito / } \\
\text { terófito }^{(3)}\end{array}$ \\
\hline Fumaria agraria Lag. ${ }^{(1)}$ & Fumariaceae & Europa $^{(1)}\left[\operatorname{med}^{(6)}\right]$ & terófito $^{(3)}$ \\
\hline Fumaria capreolata L. ${ }^{(1)}$ & Fumariaceae & Europa $^{(1)}\left[\right.$ atl-med $\left.^{(2)}\right]-$ zonas templadas $^{(2)}$ & terófito $^{(2)}$ \\
\hline $\begin{array}{l}\text { Erodium cicutarium (L.) L'Her. ex } \\
\text { Aiton }^{(1)}\end{array}$ & Geraniaceae & Mediterráneo europeo $^{(1)}\left[\right.$ med-euras $\left.^{(2)}\right]$ & $\begin{array}{l}\text { hemicriptófito / } \\
\text { terófito }^{(2)}\end{array}$ \\
\hline $\begin{array}{l}\text { Erodium moschatum (L.) L'Her. Ex } \\
\text { Aiton }^{(1)}\end{array}$ & Geraniaceae & Mediterráneo europeo ${ }^{(1)}\left[\operatorname{med}^{(2)}\right]$ & terófito ${ }^{(2)}$ \\
\hline Geranium robertianum L. ${ }^{(1)}$ & Geraniaceae & Sur de Europa ${ }^{(2)}\left[\right.$ med-smed-atl $\left.{ }^{(6)}\right]$ & $\begin{array}{l}\text { hemicriptófito } \\
\text { (terófito) }^{(2)}\end{array}$ \\
\hline Lamium amplexicaule L. ${ }^{(1)}$ & Lamiaceae & $\begin{array}{l}\text { Europa }^{(1)}[\text { euras-smed-med } \\
\text { naturalizada mundialmente } \\
\text { nata }\end{array}$ & terófito ${ }^{(2)}$ \\
\hline Papaver somniferum L. (1) & Papaveraceae & $\begin{array}{l}\text { Mediterráneo europeo }{ }^{(1)} \text { [probablemente de origen } P \text {. } \\
\left.\text { setigerum }(\text { wmed })^{(2)}\right]\end{array}$ & terófito $^{(2)}$ \\
\hline Aira caryophyllea L. ${ }^{(1)}$ & Poaceae & $\begin{array}{l}\text { Europa }^{(1)}\left[\text { subatl-smed }^{(2)}\right] \text { - zonas oceánico- } \\
\text { templadas }^{(2)}\end{array}$ & terófito $^{(2)}$ \\
\hline Avena barbata Pott ex Link ${ }^{(1)}$ & Poaceae & Europa $^{(1)}\left[\operatorname{med}^{(2)}\right]$ & terófito ${ }^{(2)}$ \\
\hline Briza minor L. ${ }^{(1)}$ & Poaceae & Europa $^{(1)}\left[\right.$ med-atl $\left.{ }^{(6)}\right]$ & terófito $^{(3)}$ \\
\hline $\begin{array}{l}\text { Bromus catharticus Vahl var. elata } \\
\text { (E.Desv.) Plachuelo }{ }^{(5)}\end{array}$ & Poaceae & Europa $^{(1)}\left[\right.$ euras-smed $\left.{ }^{(2)}\right]$ & hemicriptófito $^{(5)}$ \\
\hline Bromus hordeaceus L. ${ }^{(1)}$ & Poaceae & Europa $^{(1)}\left[\right.$ euras-smed $\left.{ }^{(2)}\right]$ & terófito $^{(3)}$ \\
\hline Bromus scoparius L. ${ }^{(1)}$ & Poaceae & Mediterráneo europeo $^{(1)}$ & terófito $^{(4)}$ \\
\hline Cynodon dactylon (L.) Pers. ${ }^{(1)}$ & Poaceae & 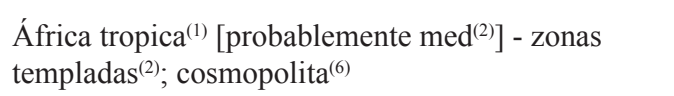 & $\begin{array}{l}\text { hemicriptófito } \\
\text { (geófito) }^{(2)}\end{array}$ \\
\hline Hordeum murinum L. s.l. ${ }^{(1)}$ & Poaceae & $\begin{array}{l}\text { Europa }^{(1)}[\text { smed-med } \\
\text { naturalizada mundialmente } \\
\text { (2) }\end{array}$ & terófito ${ }^{(2)}$ \\
\hline Rostraria cristata (L.) Tzvelev (1) & Poaceae & Europa $^{(1)}\left[\right.$ pantrop-subtrop-med $\left.{ }^{(6)}\right]$ & terófito ${ }^{(3)}$ \\
\hline Lamarckia aurea (L.) Moench ${ }^{(1)}$ & Poaceae & Europa $^{(1)}\left[\operatorname{med}^{(6)}\right]$ & terófito ${ }^{(3)}$ \\
\hline Lolium multiflorum Lam. ${ }^{(1)}$ & Poaceae & $\begin{array}{l}\text { Europa }^{(1)}\left[\text { smed-subatl }^{(2)}\right] \text { - zonas oceánico- } \\
\text { templadas }^{(2)}\end{array}$ & $\begin{array}{l}\text { hemicriptófito / } \\
\text { terófito }^{(2)}\end{array}$ \\
\hline Lolium rigidum Gaudin ${ }^{(3)}$ & Poaceae & Mediterráneo $^{(3)}\left[\right.$ euras-subocean $\left.{ }^{(6)}\right]$ & terófito $^{(3)}$ \\
\hline Poa annua L. ${ }^{(1)}$ & Poaceae & $\begin{array}{l}\text { Europa }^{(1)}[\text { no-euras-med } \\
\text { mundialmente } \\
\text { mulm }^{(2)} ; \text { cosmopolita }^{(1)}\end{array}$ & $\begin{array}{l}\text { hemicriptófito / } \\
\text { terófito }^{(2)}\end{array}$ \\
\hline Vulpia myuros (L.) C.C.Gmel. ${ }^{(1)}$ & Poaceae & Europa $^{(1)}\left[\right.$ smed-subatl $\left.^{(2)}\right]-\operatorname{cosmopolita}^{(2)}$ & terófito $^{(3)}$ \\
\hline Anagallis arvensis L. ${ }^{(1)}$ & Primulaceae & $\begin{array}{l}\text { Europa }^{(1)}[\text { euras-subocean-smed } \\
\text { zonas templadas }^{(2)} ; \text { cosmopolita }^{(6)}\end{array}$ & terófito $^{(2)}$ \\
\hline Ranunculus muricatus L. ${ }^{(1)}$ & Ranunculaceae & Europa $^{(1)}\left[\mathrm{med}^{(6)}\right]$ & terófito $^{(3)}$ \\
\hline
\end{tabular}




\begin{tabular}{|c|c|c|c|}
\hline EsPECIE & FAMILIA & $\begin{array}{l}\text { ORÍGEN GEOGRÁFICO [ELEMENTO FLORÍSTICO]- } \\
\text { EXTENSIÓN ACTUAL }\end{array}$ & FORMA DE VIDA \\
\hline Galium aparine L. ${ }^{(1)}$ & Rubiaceae & $\begin{array}{l}\left.\text { Europa }^{(1)}[\text { euras(subocean) })^{(2)}\right] \text { - zonas templadas } \\
\text { cosmopolita }^{(2)}\end{array}$ & terófito $^{(2)}$ \\
\hline Galium murale (L.) All. ${ }^{(3)}$ & Rubiaceae & Mediterráneo $^{(3)}\left[\operatorname{med}^{(6)}\right]$ & terófito $^{(3)}$ \\
\hline Galium cf. parisiense L. ${ }^{(2)}$ & Rubiaceae & Europa $^{(2)}\left[\right.$ smed-subatl $\left.{ }^{(2)}\right]$ & terófito ${ }^{(2)}$ \\
\hline Verbascum virgatum Stockes ${ }^{(1)}$ & Scrophulariaceae & Europa oriental $^{(1)}\left[\right.$ subatl $\left.^{(2)}\right]$ & hemicriptófito ${ }^{(2)}$ \\
\hline Veronica arvensis L. ${ }^{(1)}$ & Scrophulariaceae & $\begin{array}{l}\text { Europa }^{(2)}\left[\text { euras-subocean }(- \text { smed })^{(2)}\right]-\text { naturalizada } \\
\text { mundialmente } \\
(2)\end{array}$ & terófito $^{(2)}$ \\
\hline Veronica persica Poir. ${ }^{(1)}$ & Scrophulariaceae & $\begin{array}{l}\text { Suroeste asiático }{ }^{(2)} \text { - en todo el mundo con tendencia } \\
\text { de expansión subocean-smed }\end{array}$ & terófito ${ }^{(2)}$ \\
\hline Urtica urens L. ${ }^{(1)}$ & Urticaceae & $\begin{array}{l}\text { Europa }^{(1)}\left[\left(\text { no-)euras-med }{ }^{(2)}\right] \text { - zonas templadas (hasta }\right. \\
\text { cálido-templadas), naturalizada mundialmente }{ }^{(2)}\end{array}$ & terófito $^{(2)}$ \\
\hline
\end{tabular}

Fuentes:

(1) Matthei, O. 1995; (2) Oberdorfer, E. 2001; (3) Àrea De Botànica, Department De Biologia, Universitat De Les Illes Baleares: Herbari Virtual del Mediterrani Occidental. - URL: http://herbarivirtual.uib.es/cat-med/index.html [26.06.2011]; (4) TeIllier, S., J. A. FigueroA \& S. A. CASTRo 2010; ${ }^{(5)}$ URL: http://www.europeana.eu/portal/record/11616/_OPENUP_SPECIMENS_RBGE_UK_E00514317.html; ${ }^{(6)}$ Hohenester, A. \& W. Welss 1993.

\section{FORMA DE VIDA}

La forma de vida mejor representada en la flora exótica del PN La Campana es la de los terófitos, con un total de 43 especies, seguida por 11 especies que desarrollan, según las condiciones del lugar, una forma de vida terófita o hemicriptófita. Cuatro especies pertenecen a la forma de vida de los hemicriptófitos y una sola especie, Cynodon dactylon, puede aparecer eventualmente también como geófito.

Presencia de la flora exótica RUderal en las Unidades DE VEGETACIÓN

Stellaria media y Cardamine hirsuta son las especies exóticas más comunes en el Parque: están presentes en 18 y 16 muestreos respectivamente, de 21 muestreos realizados en todos los tipos de vegetación. Con la presencia en 13 de 21 muestreos también Euphorbia peplus, Vulpia myuros, Cerastium glomeratum y Galium cf. parisiense tienen muy buena representación. Avena barbata, Bromus catharticus var. elata, Carduus pycnocephalus, Conium maculatum, Hypochaeris glabra, Lamarckia aurea, Lolium rigidum, Matricaria discoidea y Trifolium tomentosum en cambio aparecen sólo en ciertos sitios.

En la Tabla III se muestra la presencia de la flora exótica ruderal en las diferentes unidades de vegetación del Parque. Las unidades que muestran una mayor presencia de especies exóticas son el Puyal con suculentas, el Matorral xerófilo y el Bosque esclerófilo. Si bien las condiciones de cobertura de estas unidades son diferentes, teniendo las dos primeras mayor disponibilidad de vacíos para el posible establecimiento de las especies exóticas y buena disponibilidad de luz, las tres unidades se encuentran entre las más transitadas por los visitantes y el ganado al interior del Parque, lo cual es una condición para la alta presencia de especies exóticas. Las mayor parte de las especies de las Tablas II y III existen como componentes esenciales en cultivos de primavera (clase Stellarietea mediae) de España y otras partes del Mediterráneo (Nezadal 1989).

TASA DE RECAMBIO A 2 y 5 M DE LOS SENDEROS

El análisis de la tasa de recambio muestra que en todas las unidades de vegetación los valores del margen del sendero a $2 \mathrm{~m}$ de distancia y en la transición de este punto a $5 \mathrm{~m}$ de distancia van bajando (Tabla IV). Esto demuestra que las especies exóticas ruderales son capaces de penetrar desde el margen del sendero hacia el terreno circundante, pero en la medida que nos alejamos del sendero, su penetración es menor. Los altos valores para el Bosque higrófilo muestran que en esta unidad sólo pocas especies son capaces de poblar el terreno también a 2 o a $5 \mathrm{~m}$ de distancia del margen del sendero. En el caso del Bosque caducifolio ya no se registró especies exóticas a $5 \mathrm{~m}$ del margen del sendero.

La Tabla IV muestra para cada unidad de vegetación las especies que se presentan con mayor frecuencia y que se extienden también desde el margen del sendero hacia el interior. 
TABLA III. Presencia de las especies exóticas ruderales en los diferentes tipos de vegetación del Parque Nacional La Campana y su frecuencia absoluta (F) en los sitios de muestreo. Entre paréntesis especies encontradas en alguno de los ingresos del Parque.

TABLE III. Presence of exotic ruderal species in each vegetation unit of La Campana National Park, including the frequency (F) of appearance in the sampling points. In brackets, species found in the park's entrance.

\begin{tabular}{|c|c|c|c|c|c|c|c|c|c|}
\hline & 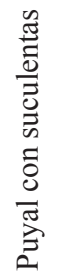 & 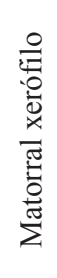 & 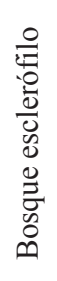 & 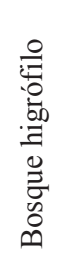 & 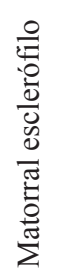 & 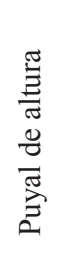 & 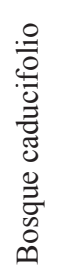 & 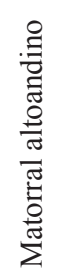 & $\mathbf{F}$ \\
\hline $\begin{array}{l}\text { Número total de especies } \\
\text { exóticas ruderales }\end{array}$ & 39 & 37 & 37 & 28 & 27 & 13 & 5 & 0 & \\
\hline Stellaria media & $*$ & $*$ & $*$ & * & $*$ & * & $*$ & & 18 \\
\hline Cardamine hirsuta & * & $*$ & * & $*$ & $*$ & * & $*$ & & 16 \\
\hline Euphorbia peplus & * & $*$ & $*$ & $*$ & $*$ & $*$ & & & 13 \\
\hline Vulpia myuros & $*$ & $*$ & $*$ & $*$ & $*$ & $*$ & & & 13 \\
\hline Cerastium glomeratum & $*$ & $*$ & $*$ & $*$ & $*$ & $*$ & & & 13 \\
\hline Galium cf. parisiense & $*$ & $*$ & $*$ & 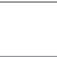 & $*$ & $*$ & & & 13 \\
\hline Trifolium glomeratum & $*$ & $*$ & $*$ & $*$ & $*$ & $*$ & & & 12 \\
\hline Geranium robertianum & $*$ & $*$ & $*$ & $*$ & $*$ & & & & 12 \\
\hline Anthriscus caucalis & $*$ & $*$ & $*$ & $*$ & & & $*$ & & 11 \\
\hline Poa annua & $*$ & $*$ & $*$ & $*$ & $*$ & & & & 11 \\
\hline Erodium cicutarium & $*$ & $*$ & $*$ & $*$ & $*$ & $*$ & & & 10 \\
\hline Galium murale & $*$ & $*$ & $*$ & $*$ & $*$ & $*$ & & & 10 \\
\hline Rostraria cristata & $*$ & $*$ & $*$ & 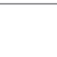 & $*$ & $*$ & & & 10 \\
\hline Hordeum murinum & $*$ & $*$ & $*$ & $*$ & $*$ & & & & 9 \\
\hline Aira caryophyllea & $*$ & $*$ & $*$ & & $*$ & & & & 9 \\
\hline Cotula australis & $*$ & $*$ & $*$ & & $*$ & & & & 9 \\
\hline Silene gallica & $*$ & $*$ & $*$ & & & * & & & 9 \\
\hline Anagallis arvensis & $*$ & $*$ & $*$ & $*$ & $*$ & & & & 8 \\
\hline Hypochaeris radicata & $*$ & $*$ & $*$ & & $*$ & $*$ & & & 7 \\
\hline Fumaria capreolata & $*$ & $*$ & $*$ & $*$ & & & & & 7 \\
\hline Urtica urens & $*$ & $*$ & $*$ & & $*$ & & & & 7 \\
\hline Anthemis cotula & $*$ & $*$ & $*$ & & $*$ & & & & 6 \\
\hline Veronica persica & $*$ & $*$ & $*$ & $*$ & & & & & 6 \\
\hline Capsella bursa-pastoris & $*$ & & $*$ & $*$ & $*$ & & & & 5 \\
\hline Trifolium dubium & & $*$ & $*$ & $*$ & $*$ & & & & 5 \\
\hline Bromus hordeaceus & $*$ & $*$ & $*$ & & $*$ & & & & 5 \\
\hline Lamium amplexicaule & $*$ & $*$ & $*$ & & & & & & 5 \\
\hline Erodium moschatum & $*$ & & $*$ & $*$ & $*$ & & & & 4 \\
\hline Medicago polymorpha & $*$ & $*$ & $*$ & $*$ & & & & & 4 \\
\hline Torilis nodosa & $*$ & $*$ & $*$ & $*$ & & & & & 4 \\
\hline
\end{tabular}


Flora exótica ruderal del Parque Nacional La Campana: HAUCK, L. ET AL.

\begin{tabular}{|c|c|c|c|c|c|c|c|c|}
\hline Ranunculus muricatus & $*$ & $*$ & $*$ & & & & & 4 \\
\hline Sonchus oleraceus & $*$ & $*$ & & $*$ & & & & 4 \\
\hline Veronica arvensis & & & $*$ & & $*$ & & & 4 \\
\hline Briza minor & & $*$ & $*$ & & & & & 4 \\
\hline Bromus scoparius & $*$ & $*$ & & & & & & 3 \\
\hline Cynoglossum creticum & & & & $*$ & $*$ & & & 3 \\
\hline Galium aparine & & & & $*$ & & & * & 3 \\
\hline Medicago arabica & & & $*$ & * & & & & 3 \\
\hline Bidens subalternans & $*$ & $*$ & & & & & & 2 \\
\hline Carthamus lanatus & $*$ & & & * & & & & 2 \\
\hline Logfia gallica & & $*$ & & & $*$ & & & 2 \\
\hline Fumaria agraria & $*$ & * & & & & & & 2 \\
\hline Polycarpon tetraphyllum & $*$ & & $*$ & & & & & 2 \\
\hline Senecio vulgaris & & & & & $*$ & $*$ & & 2 \\
\hline Sisymbrium officinale & & & $*$ & * & & & & 2 \\
\hline Avena barbata & $*$ & & & & & & & 1 \\
\hline Bromus catharticus var. elata & & & & $*$ & & & & 1 \\
\hline Carduus pycnocephalus & & & & * & & & & 1 \\
\hline Conium maculatum & & & & & & & $*$ & 1 \\
\hline Hypochaeris glabra & $*$ & & & & & & & 1 \\
\hline Lamarckia aurea & $*$ & & & & & & & 1 \\
\hline Lolium rigidum & & $*$ & & & & & & 1 \\
\hline Matricaria discoidea & & $*$ & & & & & & 1 \\
\hline Trifolium tomentosum & & & $*$ & & & & & 1 \\
\hline Cynodon dactylon & & & & & & & & (1) \\
\hline Galinsoga parviflora & & & & & & & & (1) \\
\hline Lolium multiflorum & & & & & & & & $(1)$ \\
\hline Papaver somniferum & & & & & & & & $(1)$ \\
\hline Verbascum virgatum & & & & & & & & (1) \\
\hline
\end{tabular}


TABla IV. Tasa de recambio de las especies exóticas desde el margen de los senderos a 2 y $5 \mathrm{~m}$ al interior de las unidades de vegetación.

TABLE IV. Turnover rate of exotic species from the trails to 2 and $5 \mathrm{~m}$ into the vegetation units.

\begin{tabular}{|c|c|c|c|}
\hline UNIDAD DE VEGETACIÓN & $\begin{array}{c}\mathrm{T}_{\mathrm{r}} \\
\text { MARGEN DEL SENDERO } \\
\text { DIRECTO } \\
- \\
\text { A DOS METROS DE } \\
\text { DISTANCIA DEL MARGEN } \\
\text { DEL SENDERO }\end{array}$ & $\begin{array}{c}\mathrm{T}_{\mathrm{r}} \\
\text { A DOS METROS DE DISTANCIA } \\
\text { DEL MARGEN DEL SENDERO } \\
- \\
\text { A CINCO METROS DE DISTANCIA } \\
\text { DEL MARGEN DEL SENDERO }\end{array}$ & $\begin{array}{l}\text { ESPECIES EXÓTICAS RUDERALES DE MAYOR } \\
\text { FRECUENCIA Y CAPACIDAD DE EXTENDERSE DESDE EL } \\
\text { MARGEN DEL SENDERO HACIA EL INTERIOR }\end{array}$ \\
\hline Puyal con suculentas & 0,17 & 0,64 & $\begin{array}{l}\text { Vulpia myuros, Euphorbia peplus, Silene gallica, } \\
\text { Erodium cicutarium, Galium cf. parisiense }\end{array}$ \\
\hline Matorral xerófilo & 0,23 & - & $\begin{array}{c}\text { Galium cf. parisiense, Vulpia myuros, Stellaria } \\
\text { media, Cotula australis }\end{array}$ \\
\hline Bosque esclerófílo & 0,16 & 0,29 & $\begin{array}{c}\text { Euphorbia peplus, Stellaria media, Geranium } \\
\text { robertianum, Cardamine hirsuta }\end{array}$ \\
\hline Bosque higrófilo & 0,44 & 0,71 & Geranium robertianum \\
\hline Matorral esclerófilo & 0,22 & 0,31 & $\begin{array}{c}\text { Cardamine hirsuta, Poa annua, Vulpia myuros, } \\
\text { Trifolium glomeratum }\end{array}$ \\
\hline Puyal de altura & 0,30 & 0,54 & $\begin{array}{l}\text { Vulpia myuros, Trifolium glomeratum, Silene } \\
\text { gallica }\end{array}$ \\
\hline Bosque caducifolio & 0,43 & 1 & Stellaria media, Cardamine hirsuta \\
\hline
\end{tabular}

\section{DISCUSIÓN Y CONCLUSIONES}

El presente estudio registra la presencia de 59 especies exóticas ruderales en el margen de los senderos del Parque Nacional La Campana, lo que corresponde aproximadamente a un $14 \%$ de la flora del Parque. Este es un porcentaje menor al reportado para Chile Central (27\% según Arroyo et al. 2000) y para la Cordillera de la Costa de la Región de Valparaíso (un 34\% según Teillier et al. 2010). Ello implica que a pesar de la presencia de especies exóticas en su interior, el Parque Nacional sigue siendo, en comparación con otros sitios de la región, un área relativamente menos intervenida. El predominio de las especies eurasiático-norteafricanas en Chile central se debe en gran parte al fuerte (y traumático en muchos sentidos) contacto inicial entre Chile y el Viejo Mundo (Arroyo et al. 2000). Al examinar las especies de origen eurasiático-norteafricanas se aprecia que entre ellas se encuentran muchísimas especies mediterráneas. Este hecho se debe a su capacidad de adaptación a las condiciones climáticas en Chile central, en consonancia con las condiciones de su lugar de origen (Casado et al. 2015). De hecho las especies de la región mediterránea son reconocidas por su alta capacidad invasora. Hay autores que consideran esta característica el resultado de un proceso evolutivo: como el origen de la agricultura prehistórica está en la región en torno al Mar Mediterráneo, la modificación antropogénica promueve desde hace más de cinco milenios los procesos de especiación (Richter 2001). Es también esperable que en la flora exótica ruderal domine la forma de vida de terófitos, lo cual es por lo demás una característica de la flora exótica de la provincia de Valparaíso (Teillier et al. 2010), y de Chile Central (Arroyo et al. 2000).

Debido a la mayor presencia de especies exóticas en las unidades de carácter xerófilo o esclerófilo, al parecer existen dos factores que influyen considerablemente en el éxito de plantas introducidas. El primer factor es la luz disponible y el segundo es el nivel altitudinal. La cantidad de plantas exóticas ruderales fue claramente más alta en los muestreos que se realizaron en senderos al interior de puyales o matorrales xerófilos, de menor cobertura relativa. De hecho, en sitios abiertos de la Cordillera de la Costa, se ha reportado que las especies exóticas pueden alcanzar una cobertura superior al 70\% (Teillier et al. 2010).

Figueroa et al. (2004) describen una baja tolerancia de la flora exótica anual a la sombra. La escasa luz disponible en este caso en el Bosque higrófilo parece influir en la menor cantidad de especies exóticas en los márgenes de los senderos y su extensión desde los márgenes hacia el interior. La razón para esto se debe a que este tipo de vegetación muestra un grado de cobertura alto (entre el 80 y $100 \%$ ). La cantidad de luz disponible está directamente relacionada con el cubrimiento por las copas de los árboles y el ancho del sendero. 
En el Parque también se aprecia que las especies exóticas están principalmente bajo los $1.100 \mathrm{~m}$ de altitud. Las condiciones ambientales vinculadas con la altitud dificultan o impiden totalmente el establecimiento de las especies exóticas ruderales. En general, la época vegetativa se acorta por cada $100 \mathrm{~m}$ de altitud aproximadamente en una semana (Frey \& Lösch 1998). Aparte de eso, la radiación en las montañas alcanza valores mucho más altos que en los valles aumentando sobre todo la radiación ultravioleta. La temperatura del aire y de las capas del suelo más profundas desciende con cada $100 \mathrm{~m}$ de altura aproximadamente 0,6 grados. También la precipitación en forma de nieve y la frecuencia e intensidad del viento aumentan en altitud (Frey \& Lösch 1998). El fenómeno de una menor presencia de especies exóticas a mayor altitud ha sido ampliamente documentado en distintas regiones del planeta: a mayor altitud se produce un efecto filtro para las especies exóticas, por las condiciones ambientales más adversas ya mencionadas (Pauchard \& Alaback 2004, Alexander et al. 2011, Seipel et al. 2012). A ello se suma en el caso de La Campana una menor densidad de senderos y un tránsito más escaso de visitantes y ganado a mayor altitud. Ello resulta en una tasa de implantación de semillas exóticas menor a mayor altitud.

El potencial de invasibilidad de especies exóticas en altura podría modificarse en relación con el cambio climático global. Existe mucha documentación en distintas partes del mundo acerca de los cambios en la fenología y capacidad de dispersión que estarían afectando cada vez más ambientes de altura y específicamente áreas protegidas de montaña (Pauchard et al. 2009, 2016).

Ello plantea importantes desafíos para el manejo de este tipo de áreas silvestres protegidas, pues se trata de fenómenos globales que se expresan a escala local (Kueffer et al. 2013). Entre las medidas más obvias se encuentra la información y capacitación de la población local en relación con el potencial invasor de ciertas especies, así como el control específico del ganado, la actividad turística, y la flora de jardines del entorno del área protegida (Kueffer et al. 2013). En este sentido, en Chile se ha avanzado relativamente poco tanto en el control como en la difusión de los problemas asociados a las especies invasoras (McDougall et al. 2011).

En el caso de La Campana, es conveniente diseñar un programa de monitoreo de las especies exóticas. Aunque esto dependa de los escasos recursos de la administración del Parque, conviene enfocar la atención tanto en las especies que se encuentran ya en un estado avanzado de integración en la vegetación del Parque, como en aquellas cuya frecuencia es aún menor.

Parece sensato asumir la propuesta de Arroyo et al. (2000) de observar especialmente la extensión de las especies ya conocidas como "agresivas". De acuerdo con el ISSG (Invasive Species Specialist Group), un equipo de la UICN (Unión Internacional para la Conservación de la Naturaleza), este sería el caso de las siguientes especies exóticas registradas en el Parque: Hypochaeris radicata, Senecio vulgaris, Sonchus oleraceus, Stellaria media, Trifolium dubium, Erodium cicutarium, Cynodon dactylon y Poa annua (ISSG 2015). Aquellas especies reconocidas como altamente invasoras y ya naturalizadas en áreas protegidas deben ser incorporadas en programas de prevención, control y erradicación, y pasar a formar parte de un Programa Nacional Integrado para la Gestión de las Especies Exóticas Invasoras (Fuentes \& Pauchard 2011), con la participación activa de SAG (Servicio Agrícola Ganadero). La dimensión con la que las especies nativas o los ecosistemas al interior del Parque están siendo o serán perjudicadas por la flora exótica, y una reacción adecuada a posibles invasiones biológicas, requiere monitoreo de largo plazo y el control de la acción de los visitantes y el ganado ocasional, para lo cual el organismo a cargo de la administración de la unidad (en este caso CONAF), debería contar con los recursos y el personal de operación necesarios.

\section{AGRADECIMIENTOS}

ACONAF Región de Valparaíso, en especial al administrador del Parque Nacional La Campana don Christian Díaz y a todos los guardaparques por la cooperación, el apoyo y la literatura que nos facilitaron. A la Sra. Mélica Muñoz Schick por su amable apoyo con la identificación de las especies en el herbario del Museo Nacional de Historia Natural en Santiago (SGO). Un fuerte agradecimiento también a la Sra. Gloria Rojas Villegas por facilitar el acceso al Herbario SGO. Kevin Bartke realizó la traducción desde el alemán. Marcelo Leguía realizó la cartografía. Agradecemos también los aportes de dos revisores anónimos que han mejorado sustancialmente el trabajo. Fondecyt $N^{\text {os }} 11085016$ y 1150425 .

\section{REFERENCIAS}

Alexander, J.M., C. Kueffer, C. Daehler, P.J. Edwards, A. Pauchard, T. Seipel \& Miren consortium. 2011. Assembly of non-native floras along elevational gradients explained by directional ecological filtering. Proceedings of the National Academy of Sciences 108: 656-661.

Àrea De Botànica, Department De Biologia, Universitat De Les Illes Baleares. 2014. Herbari Virtual del Mediterrani Occidental. -URL: http://herbarivirtual.uib.es/cat-med/ index.html [26.06.2011]

Arroyo, M.T. K., C. Marticorena, O. Matthei \& L. Cavieres. 2000. Plant Invasions in Chile: Present Patterns and Future Predictions. In: H.A. Mooney \& R. J. Hobbs (eds.) Invasive Species in a Changing World. pp. 385-421. Island Press, Washington.

Casado, M.A., B. Acosta-Gallo, L. Sánchez-Jardón, I. MartínForés, I. Castro, C. Ovalle, A. del Pozo \& J.M. De 
Miguel. 2015. Interactive effects of source and recipient habitats on plant invasions: distribution of exotic species in Chile. Diversity and Distributions 21: 609-619.

CAstro, S. \& M. MuÑoz-SCHICK. 2006. Compuestas naturalizadas en Chile: importancia de la flora exótica como agente del cambio biótico. Revista Chagual (Jardín Botánico de Santiago) 4: 29-38.

Cowling, R.M., P.W. Rundel, B.B. Lamont, M.T.K Arroyo \& M. Arianoutsou. 1996. Plant diversity in mediterraneanclimate regions. Trends in Ecology and Evolution 11: 362366.

Davis, S.D., V.H. Heywood, O. Herrera-MacBryde, J. VillaLobos \& A.C. Hamilton. 1997. Centres of Plant Diversity: A Guide and Strategy for their Conservation. Vol. 3: The Americas. IUCN Publ. Unit, Cambridge. 562 pp.

Elórtegui, S. \& A. Moreira-MuÑoz (eds.). 2009. Parque Nacional La Campana: Origen de una Reserva de la Biosfera en Chile central. (2a ed.) Taller La Era, Viña del Mar. 176 pp.

Figueroa, J.A., S.A. Castro, P.A. Marquet \& F.M. Jaksic. 2004. Exotic plant invasions to the Mediterranean region of Chile: causes, history and impacts. Revista Chilena de Historia Natural 77: 465-483.

Frey, W. \& R. LÖSCH. 1998. Lehrbuch der Geobotanik. Pflanze und Vegetation in Raum und Zeit. G. Fischer, Stuttgart. $436 \mathrm{pp}$.

Fuentes, N. \& A. Pauchard (eds.). 2011. Documento Técnico sobre el Taller: Evaluación de Riesgo de especies de Plantas Introducidas (ERPI) en Chile: estableciendo prioridades para su manejo o control. Laboratorio de Invasiones Biológicas, Universidad de Concepción. 21 pp.

Fuentes, N., A. Pauchard, P. Sánchez, J. Esquivel \& A. Marticorena. 2013. A new comprehensive database of alien plant species in Chile based on herbarium records. Biological Invasions 15: 847-858.

GaJARDo, R. 1994. La vegetación natural de Chile. Clasificación y distribución geográfica. Editorial Universitaria, Santiago. $165 \mathrm{pp}$.

Gardener, M., R. Bustamante, I. Herrera, G. Durigan, V. Pivello, M. Moro, A. Stoll, B. Langdon, Z. Baruch, A. Rico, A. Arredondo-NuÑEZ \& S. Flores. 2011. Plant invasion in Latin America: fast track to a more focused agenda. Plant Ecology \& Diversity 5(2): 225-232.

Geomanejo Consultores. 2012. Catastro Flora y Fauna Parque Nacional La Campana: Identificación Zonas de Conservación. Documento inédito, insumo nuevo Plan de Manejo Parque Nacional La Campana, CONAF Región de Valparaíso. 62 pp.

Hoffmann, A. 1998. Flora silvestre de Chile. Zona central. Una guía para la identificación de las especies vegetales más frecuentes. Fundación Claudio Gay, Santiago de Chile. $255 \mathrm{pp}$.

Hohenester, A. \& W. Welss.1993. Exkursionsflora für die Kanarischen Inseln. Ulmer, Stuttgart. 374 pp.

ISSG (Invasive Species Specialist Group of IUCN). 2015. Global Invasive Species Database. - URL: http://www.issg.org/ database/welcome [13.07.2015].

JAKsIC, F. \& S. CASTro (eds.) 2014. Invasiones Biológicas en Chile: Causas globales e impactos locales. Ediciones UC, Santiago. 528 pp.

Jiménez, A., A. Pauchard, L.A. Cavieres, A.E. Marticorena \&
R.O. Bustamante. 2008. Do climatically similar regions contain similar alien floras? A test from the Mediterranean areas of Chile and California. Journal of Biogeography 35: 614-624

Jiménez, A., A. Pauchard, A. Marticorena \& R.O. Bustamante. 2013. Patrones de distribución de plantas introducidas en áreas silvestres protegidas y sus áreas adyacentes del centro-sur de Chile. Gayana Botánica 70(1): 87-97.

Kowarik, I. 2003. Biologische Invasionen. Neophyten und Neozoen in Mitteleuropa. Ulmer, Stuttgart. 380 pp.

Kueffer, C., K. McDougall, J. Alexander, C. Daehler, P. Edwards, S. Haider, A. Milbau, C. Parks, A. Pauchard, \& Z. A. Reshi. 2013. Plant invasions into mountain protected areas: assessment, prevention and control at multiple spatial scales. In: L.C. Foxcroft, D.M. Richardson, P. Pysek \& P. Genovesi (eds.), Alien Plant Invasions in Protected Areas: A Global Assessment. Springer Series in Invasion Ecology 7: 89-113; Springer Netherlands.

Luebert, F., M. Muñoz-Schick \& A. Moreira-Muñoz. 2009. Vegetación y Flora. En: S. Elórtegui \& A. Moreira-Muñoz (eds.), Parque Nacional La Campana: Origen de una Reserva de la Biosfera en Chile central. (2a ed.), pp. 3669. Taller La Era, Viña del Mar, Chile.

Mardones, D., N. Fuentes \& A. Saldaña. 2015. Comparación de la severidad de invasión asociada a caminos con diferentes niveles de perturbación en un bosque templado lluvioso del sur de Chile. Gayana Botánica 72(2): 221-230.

Matthei, O. 1995. Manual de las malezas que crecen en Chile. Alfabeta Impresores, Santiago de Chile. 545 pp.

McDougall, K.L., A.A. Khuroo, L. L. Loope, C.G. Parks, A. Pauchard, Z.A. Reshi, I. Rushworth \& C. Kueffer. 2011. Plant Invasions in Mountains: Global Lessons for Better Management. Mountain Research and Development 31: 380-387.

Moreira-Muñoz, A. \& A. Salazar. 2014. Reserva de la Biosfera La Campana-Peñuelas: micro-región modelo para la planificación del desarrollo regional sustentable. En: A. Moreira-Muñoz \& A. Borsdorf (eds.), Reservas de la Biosfera de Chile: Laboratorios para la Sustentabilidad. Academia de Ciencias de Austria, Vol. 17: 104-121. Instituto de Geografía UC, Santiago, serie Geolibros.

Moreira-Muñoz, A. \& J. Troncoso. 2014. Representatividad Biogeográfica de las Reservas de la Biosfera de Chile. En: A. Moreira-Muñoz \& A. Borsdorf (eds.), Reservas de la Biosfera de Chile: Laboratorios para la Sustentabilidad. Academia de Ciencias de Austria, Vol. 17: 24-61. Instituto de Geografía UC, Santiago, serie Geolibros.

Mittermeier, R.A., P. Robles-Gil, M. Hoffmann, J.D. Pilgrim, T.B. Brooks, C.G. Mittermeier, J.L. Lamoreux \& G.A.B. FonsECA. 2004. Hotspots Revisited: Earth's Biologically Richest and Most Endangered Ecoregions. CEMEX. Ciudad de México, 390 pp.

Navas, L. E. 1973. Flora de la cuenca de Santiago de Chile. Tomo I: Pteridophyta, Gimnospermae, Monocotyledoneae. Ediciones de la Universidad de Chile, Santiago de Chile. $301 \mathrm{pp}$.

Navas, L. E. 1976. Flora de la cuenca de Santiago de Chile. Tomo II: Dicotyledoneae, Archichlamydeae. Ediciones de la Universidad de Chile, Santiago de Chile. 559 pp.

Navas, L. E. 1979. Flora de la cuenca de Santiago de Chile. Tomo 
III. Ediciones de la Universidad de Chile, Santiago de Chile. 509 pp.

Newsome, A.E. \& I.R. Noble. 1986. Ecological and physiological characters of invading species. In: R.H. Groves \& J. J. Burdon (eds.), pp. 1-20. Biological Invasions Cambridge. Cambridge University Press.

NeZADAL, W. 1989. Unkrautgesellschaften der Getreide- und Frühjahrs-Hackfruchtkulturen (Stellarietea mediae) im mediterranen Iberien. Dissertationes Botanicae 143. Berlin, Stuttgart. 205 pp.

OBerdorfer, E. 2001. Pflanzensoziologische Exkursionsflora für Deutschland und angrenzende Gebiete. Ulmer, Stuttgart (Hohenheim). 1051 pp.

Pauchard, A. \& P. B. Alaback. 2004. Influence of Elevation, Land Use, and Landscape Context on Patterns of Alien Plant Invasions along Roadsides in Protected Areas of SouthCentral Chile. Conservation Biology 18: 238-248.

Pauchard, A., C. Kueffer, H. Dietz, C.C. Daehler, J. Alexander, P.J. Edwards, J.R. Arévalo, L.A. Cavieres, A. Guisan, S. Haider, G. Jakobs, K. McDougall, C.I. Millar, B.J. Naylor, C.G. Parks, L.J. Rew, \& T. Seipel. 2009. Ain't no mountain high enough: plant invasions reaching new elevations. Frontiers in Ecology and the Environment 7: 479-486.

Pauchard, A., C. Quiroz, R. García, C.H. Anderson \& M.T.K. Arroyo. 2011. Invasiones biológicas en América Latina y el Caribe: tendencias en investigación para la conservación. En: J. Simonetti \& R. Dirzo (eds.), Conservación Biológica: Perspectivas desde América Latina. pp. 79-94. Editorial Universitaria, Santiago, Chile.

Pauchard, A., N. Fuentes, A. Jiménez, R. Bustamante \& A. Marticorena. 2013. Alien Plants Homogenise Protected Areas: Evidence from the Landscape and Regional Scales in South Central Chile. In: L.C. Foxcroft, D.M. Richardson, P. Pysek \& P. Genovesi (eds.), Plant Invasions in Protected Areas: Patterns, Problems and Challenges. Springer Series in Invasion Ecology 7: 191-208. Springer, Netherlands.

Pauchard, A., A. Milbau, A. Albihn, A. Alexander, T. Burgess, C. Daehler, G. Englund, F. Essl, B. Evengård et al. 2016. Non-native and native organisms moving into high elevation and high latitude ecosystems in an era of climate change: new challenges for ecology and conservation. Biological Invasions 18(2): 345-353.

Pliscoff, P. 2009. Climatología. En: Elórtegui, S. \& A. Moreira Muñoz (eds.), Parque Nacional La Campana: Origen de una Reserva de la Biosfera en Chile central. (2a ed.), pp. 22-26. Taller La Era, Viña del Mar, Chile.

Quiroz, C.L., A. Pauchard, L.A. Cavieres \& C.B. Anderson. 2009. Análisis cuantitativo de la investigación en invasiones biológicas en Chile: tendencias y desafíos. Revista Chilena de Historia Natural 82: 497-505.
RAy, C.A., V. Escobedo-Echeverría, S.A. CAStro \& F.M. JaKsic. 2014. Catálogo de la flora y fauna invasora en Chile continental e insular oceánico. En: F. Jaksic \& S. Castro (eds.), Invasiones Biológicas en Chile: Causas globales e impactos locales. pp. 491-505. Ediciones UC, Santiago, Chile.

ReIche, C. 1903. Las malezas que invaden a los cultivos de Chile y el reconocimiento de sus semillas. Imprenta FrancoChilena, Santiago, Chile. 88 pp.

Richter, M. 2001. Vegetationszonen der Erde. Klett-Perthes, Gotha. 448 pp.

SAX, D.F. 2002. Native and naturalized plant diversity are positively correlated in scrub communities of California and Chile. Diversity and Distributions 8: 193-210.

Seipel, T., C. Kueffer, L. Rew, C. Daehler, A. Pauchard, B. Naylor, J.M. AleXander, C. Parks, P.J. Edwards, J.R. Arevalo, L.A. Cavieres, H. Dietz, G. Jakobs, K. McDougall, R. Otтo \& N. Walsh 2012. Processes at multiple scales affect non-native plant species richness and similarity in mountains around the world. Global Ecology and Biogeography 21: 236-246.

Teillier, S., R. Rodríguez \& M.T. Serra. 2003. Lista preliminar de plantas leñosas, alóctonas, asilvestradas en Chile Continental. Chloris Chilensis año 6, $\mathrm{n}^{\mathrm{o}} 2$. http://www. chlorischile.cl.

Teillier, S., J.A. Figueroa \& S.A. Castro. 2010. Especies exóticas de la vertiente occidental de la Cordillera de la Costa, Provincia de Valparaíso, Chile central. Gayana Botánica 67(1): 27-43.

TrEmp, H. 2005. Aufnahme und Analyse vegetationsökologischer Daten. Ulmer, Stuttgart. $141 \mathrm{pp}$.

Vanderplank, S.E., A. Moreira-Muñoz, C. Нobohm, G. Pils, J. Noroozi, V.R. Clark, N.P. Barker, W. Yang, J. Huang, K. Ma, C.Q. Tang, M.J.A.Werger, M. Ohsawa \& Y. YANG. 2014. Endemism in Mainland Regions - Case Studies: [Central Chile Ecoregion]. In: C. Hobohm (ed.), Endemism in Vascular Plants. Series Plant and Vegetation 9: 205-308. Springer, Dordrecht.

VIDAL, O. 2005. Flora exótica adyacente a senderos remotos en el Parque Nacional "Torres del Paine" (Magallanes, Chile). Tesis de Grado presentada como parte de los requisitos para optar al Grado de Licenciado en Ciencias Biológicas. Facultad de Ciencias, Universidad Austral de Chile.

VILLASEÑOR, R. 1998. Guía para el reconocimiento de las especies arbóreas y arbustivas en el Parque Nacional La Campana. Editorial Universidad de Playa Ancha, Valparaíso. 207 pp.

Williamson, M. 1996. Biological Invasions. Chapman and Hall, New York. 244 pp.

Weber, E. \& J. R. Joshi. 2009. Biologische Invasionen: Mechanismen, Auswirkungen, Chancen und Risiken. Koprint, Zürich. 74 pp.

Recibido: 20.01.16

Aceptado: 01.08.16 